\title{
Representación de conceptos: rasgos y esquemas
}

\section{Herminia Peraita*}

\author{
Universidad Nacional de Educacion a Distancia
}

\section{INTRODUCCION}

El presente trabajo se propone establecer una comparación a nivel teórico entre las caracteristicas de ciertas estructuras de conocimiento o, mejor dicho, de representación del conocimiento de la vida diaria, que suelen abordarse por separado, como estructuras diferentes, y que una consideración más pormenorizada de las mismas nos hace ver como muy semejantes. Nos estamos refiriendo, por una parte, al concepto de guión - script-, tipo de esquema, tal y como lo definen Schank y Abelson, 1977; Rumelhart y Ortony, 1977; Abelson, 1981, y Nelson, 1983, que representa hechos o acontecimientos de la vida diaria, y, por otra parte, a las categorías naturales de Rosch, 1976; Rosch y Mervis, 1975, y Mervis y Rosch, 198 I, que representan objetos también de la vida diaria.

Se trata de hacer un análisis de tipo teórico, estructural, seguido de uno empírico, llevado a cabo sobre datos obtenidos de muestras de sujetos, que nos permitan establecer una analogía entre las características y propiedades del guión a esquema, del tipo - ir al restaurante, ir al dentista, a un funeral, etc.- y las de las categorias naturales y sociales que representan objetos y personas -árbol, pez, vehículo, instrumento musical, etc.

Se trata, por tanto, de aproximar dos enfoques que según algunos autores deben ocupar áreas distintas en el campo de la representación conceptual (Scholnick, 1983) y, por tanto, están en competencia, siendo ambas alternativas distintas a la forma piagetiana de representación de los conceptos.
$\mathrm{Ni}$ siquiera creo que deban ser consideradas como áreas convergentes (Nelson, 1983; De Vega, 1984), sino uno e idéntico campo de investigación, aunque, lógicamente, el psicólogo evolutivo, e] cognitivo y el teórico de I. A. (inteligencia artificial) aborden este campo, en principio, desde perspectivas distintas.

Voy a considerar el guión -y el esquema en general - como una categoría con una estructura interna y una organización jerárquica y las categorias naturales como guiones o esquemas.

\section{PLANTEAMIENTO GENERAL: PREGUNTAS BASICAS}

Las preguntas que surgen ante los tipos de representación que tratamos de analizar son (Scholnick, 1983 ):

¿Implican realmente dos tipos de operaciones de procesos, dos procesadores cognitivos diferentes, dos aspectos informativos diferentes de la realidad? ¿Cuál de las formas es más básica para el modo de pensar humano?

Voy a defender el punto de vista de que son iguales. Ambas unidades cognitivas implican conocimiento categórico, ambas representan categorías (de objetos, personas y acontecimientos) mediante sustitutivos equivalentes $y$, aunque a primera vista la diferencia esencial es que son modelos distintos de representación, pues uno se centra en acciones, secuencias, movimientos y procesos - hechos dinámicos en una palabra- y el otro en los objetos en sí y en los atributos que los componen, la revisión empirica y posterior a análisis de ciertos datos nos indica

* Dirección de la autora: Universidad Nacional de Educación a Distancia, Facultad de Psicología, Ciudad Universitaria. 28023 Madrid. 
que no existe tal dicotomía o, al menos, de manera tan radical que nos haga pensar en diferentes operaciones, procesadores y aspectos de la realidad, sino que las representaciones conceptuales están impregnadas de ambos aspectos: en representaciones de categorías naturales y de personas, supuestamente estáticas -un árbol, un águila, un pantalón, un médico-, encontramos información dinámica $y$ relacional, $y$ en representaciones de acciones o hechos - ir al dentista, ir a un funeral, a un restaurante--, encontramos, por fuerza, rasgos y atributos estáticos sobre las personas y objetos a que hacen referencia, si bien, no tiene por qué ser siempre de manera explicita.

La indeterminación o ausencia de especificación que encontramos en Rosch respecto al formato o tipo de código representacional - si imágenes, preposiciones, etcétera- para las categorías naturales hacen que uno pueda inclinarse por cualquiera de ellos o por ninguno, ni más ni menos que sucede con los guiones. $\mathrm{El}$ formato parece ser proposicional, pero nada hay en contra de las imágenes. Lo que sucede es que Rosch explicita mucho más las características del mundo externo, las claves de validez que éste nos proporciona para estructurarlo - clusters de atributos, covariación entre los mismos, etc.-, pero deja sin especificar el formato; en los teóricos del guión o script sucede justo lo contrario: el mundo externo sobre el que éstos se originan es menos analizado mientras que los modelos representacionales se detallan mucho más, entre otras cosas, porque su aplicación a campos que exigen mayor grado de formalización -I. A.- así lo requiere.

\section{Naturaleza esquemática}

En principio, y en virtud de una ley de economía imprescindible en cualquier caracterización de procesos cognitivos, las representaciones mentales, sean de objetos naturales o artificiales, de acontecimientos, de gramáticas, etc., han de ser esquemáticas por naturaleza. Sería imposible de lo contrario poder estructurar toda la cantidad de información que nos rodea; para poder manejar esta información hay que reducirla a proporciones que permitan esa manipulación de la misma, tanto a nivel cognitivo como con- ductual (Rumelhart y. Ortony, 1977, y Rosch, 1978).

Se impone, pues, al sujeto, como un primer paso, hacer cortes y establecer limites, clasificar, en una palabra, la realidad que nos circunda y fruto de ese proceso clasificador, categorizador, estructurador, son tanto los guiones esquemáticos de hechos que nos proponen Schank, Abelson, Nelson, Rumelhart y Ortony, Minsky, etc., como las categorías naturales de Rosch. La representación conceptual implica siempre la categorización, sea cual fuere su finalidad y objetivos (Scholnick, 1983).

\section{Diacronía-sincronía}

Categorias y guiones.-Ambos son estructuras abstractas de conocimiento, verdaderas unidades de organización de la memoria - nos referimos a semántica, no a episódica, si es que hay dos-; es más, ambas son tipos de esquemas pero el guión "contiene una secuencia más o menos estándar de hechos que caracterizan actividades típicas realizadas por per, sonas - u objetos - en unos lugares, con unos medios y con unos fines", etc. (Schank y Abelson, 1977), y este tipo de información que contiene el guión -que, por cierto, como es bastante obvio, está muy determinada por el contexto de donde se extrae o abstrae: no es lo mismo «tomar el té» en Japón que en Inglaterra, que en España-, está organizado, fundamentalmente, para algunos autores, en torno a la dimensión tiempo; representa una realidad que tiene su razón de ser diacrónicamente, que se desarrolla en un tiempo. Para otros, sin embargo, el efecto de orden temporal es secundario a la "centralidad». Existe en la realidad, pero en las representaciones no es primordial. Por lo mismo, el procesamiento de un guión no requiere búsqueda secuencial lineal (Rumelhart y Ortony, 1977).

Si consideramos por un momento el contenido de un guión como un objeto, esto es, sincrónicamente, la estructura es la misma que la de una categoría natural: los hechos parciales - «events»- pasan a ser rasgos o características o propiedades de la situación central, del guión, siendo unos más o menos representativos que otros en función de criterios de frecuencia, como luego vieremos. 


\section{Estructura interna}

\section{Limites borrosos y tipicidad}

La definición del guión como «estructura relacional que representa objetos, por sus propiedades y funciones y por su lugar en redes de acontecimientos o jerarquías semánticas», es válida para ambos porque los dos incluyen conocimiento enciclopédico de propiedades, funciones y contextos.

El tipo de relaciones primitivas inherentes al esquema -agente, efecto, causa, lugar, etc. - los encontramos también en toda representación categórica de un objeto (Peraita, 1984, y en prensa).

El guión posee, además, idéntica estructuración prototípica que la de las categorias naturales y predictibilidad de atributos dentro de una categoría. De la misma manera que conocemos, dado un determinado guión, cuál es el actor, actividad, lugar, etcétera, que mejor llena un determinado hueco - "Gap Filling" - lo sabemos también en el caso de una categoría natural; pongamos por caso «árbol», categoría de la que nos dan todo tipo de información, excepto la correspondiente a es un... (clase) y tiene... (propiedades) (Galambos y Rips, 1982).

Barsalou (1983) hace unas predicciones semejantes al referirse a las «categorias ad boc», definiéndolas como categorías instrumentales que la gente construye para determinados fines. Ejemplo: «cosas para vender en el mercado de segunda mano"; «trajes que se pueden llevar a determinado tipo de fiestas - punk-, por ejemplo»; "maneras de hacer amigos», etc. Estas categorias poseen cierto paralelismo en su estructura interna en lo que respecta a los gradientes de tipicidad.

Cuando Rumelhart y Ortony (1977) nos dicen, hablando de las variables de los esquemas, en concreto de sus limitaciones (restricciones selectivas en el léxico de la semántica) que "no suelen ser absolutas» y que "es raro el caso en que una variable no pueda nunca aceptar un valor de un cierto tipo", ¿qué otra cosa nos están diciendo más que los límites de las categorías $y$, por tanto, la pertenencia de un objeto a la misma, no son cuestión de todo o nada, sino que son más bien borrosos, y algunos objetos más típicos que otros? (Normalmente, romper implica un objeto material, pero se puede "romper una amistad, una fe, un afecton, etc.)

Esta flexibilidad en las limitaciones de las variables como característica fundamental de los esquemas, que aclara la diferencia entre definición (en sentido clásico) y conocimiento, y permite representar el conocimiento de manera flexible, reflejando la tolerancia humana a la vaguedad e imprecisión (Rumelhart y Ortony, 1977), es la misma que manifiesta Rosch al considerar los límites borrosos de las categorias (Barsalou, 1981 ; MedinSmith, 1984).

\section{Formación-desarrollo}

$¿$ Es diferente el proceso que subyace al desarrollo de las categorías y al de los guiones? ¿Es la «representación de sucesos" el germen u origen de la vida conceptual como manifiestan algunos autores? (Nelson, 1983). Aunque así fuera -lo cual es difícil de demostrar, pues el recurso al cambio asociativo sintagmático-paradigmático, no nos parece sea suficientemente explicativo-, el proceso parece ser más bien dual y simultáneo; el niño, probablemente, extrae - abstrae clases y categorias de las representaciones de sucesos concomitantemente, por lo cual, si esto es así, la representación de un acontecimiento reiterado de su entorno, permite y potencia la formación - y representación - de una o varias clases o/y categorías de objetos, personas, etc.

El proceso que subyace al desarrollo de categorias no tiene por qué ser diferente al que subyace al desarrollo de "events». Ambas estructuras requieren explicación a partir de unos contextos determinados. En las dos están implicadós idénticos procesos cognitivos -atención, percepción, memoria $y$, por supuesto, lenguaje-. Ambos suponen estabilización mental de una realidad dinámica y cambiante, pero nunca estabilización definitiva, por lo cual la descontextualización y estabilización que Nelson supone para las categorías semánticas y naturales nunca llega a ser total. Ambos están formados a partir de experiencias pasadas y abstracciones hechas a partir de las mismas y la parte de "construcción" por parte del sujeto que coordina esas experiencias pasadas que hay en el guión la hay en la categoría también. 


\section{Estructura externa}

\section{Organización jerárquica}

Tanto los guiones - y esquemas, en general - como las categorías naturales, físicas y sociales presentan una organización jerárquica, lo cual implica diversos niveles de generalidad o abstracción $y$ ejemplos o miembros de la categoría situados en cada uno de los niveles en virtud de su mayor o menor número de especificaciones o matizaciones, expresadas a través de atributos cada vez más ligados a situaciones concretas. El equivalente de las "categorías supraordenadas» de la teoría de Rosch son los "metascripts» de Abelson, así como los «tracks»-caminos o sendas - equivalen, funcional y estructuralmente, a las «categorías subordinadas"».

Las categorías supraordenadas -mueble-, situadas a un nivel de abstracción mayor que las de nivel básico, tienen muy pocos atributos en común con otros elementos de la categoria - silla, mesa, cama-, mientras que las categorías subordinadas - silla de cocina - comparten la mayor parte de los atributos con elementos de la categoria - silla de playa- de comedor, de estilo; etc. (Rosch, 1976).

La semejanza de este análisis con el que efectúan Shank y Abelson (1977) y Abelson ( $198 \mathrm{I}$ ) al analizar las modificaciones o desviaciones de los guiones, y en concreto una de ellas, los «tracks» que, en lo que se refiere al guión «ir al restaurante», supone la posibilidad de «ir a un restaurante convencional», "a uno chino", "a un "drugstore"», etc., con sus especificaciones pertinentes, la semejanza, digo, no puede ser más obvia. Lo mismo sucede con los «metascripts» (Abelson, i972) o esquemas dominantes y los «subframes" y "superframes» de Minsky (1972). La función del nivel básico de categorización es la economía de las representaciones conceptuales. La estructuración o configuración del mundo circundante en distintos niveles de categorización no será idéntica para la persona con un conocimiento exhaustivo de un campo dado que para el novicio. Pensemos por un momento lo que implica el conocimiento asignado a un avión —atributos, coordinación de movimientos que implica el manejo, etc.- por un técnico o por el hombre de la calle. De la misma manera tanto las categorías más básicas en cuanto más ligadas a lo físiológico y físico - color $y$ forma - como las de objetos concretos y sociales, se estructuran en función de una lógica categorial universal -no así, lógicamente, en cuanto a los contenidos(Rosch, 1978) en torno a prototipos.

\section{Aspectos funcionales}

El objetivo principal de la presente investigación fue, resumiendo, establecer una comparación en una serie de parámetros o aspectos estructurales -naturaleza esquemática, eje diacrónico-sincrónico, estructura interna, formación y desarrollo y organización jerárquica - y nos limitaremos a afirmar cómo el carácter plurifuncional de los esquemas es compartido por las categorías naturales y sociales.

Tanto los procesos de comprensión del lenguaje, como de percepción visual, memoria de textos y narraciones, organización de la conducta, generación de expectativas, etc., son facilitados, potenciados e, incluso, determinados en función del conjunto de esquemas del sujeto. La versatilidad de dicho concepto lo hace aplicable tanto a estímulos visuales como a situaciones, personas, roles, etc., y dada la consideración que hemos otorgado a la categoria natural y social como esquema del mundo natural o de aspectos del mismo, por fuerza ha de compartir dichas caracteristicas funcionales.

\section{Experimento}

Sujetos

Se ha trabajado con sujetos de $2 .^{\circ}, 4 .^{\circ}$ y $70^{\circ}$ de E.G.B. del Colegio Nacional Nicaragua, de Madrid, siendo el $\mathrm{N}$ de cada uno de los tres grupos, respectivamente, 25,25 y 25 .

\section{Material}

Se entregó a cada uno de los niños una baraja de 20 fichas en cuyo ángulo superior derecho estaba escrito un nombre que indicaba una categoría socio-profesional, siendo el orden de presentación aleatorio para cada sujeto. Los nombres de dichas categorias pertenecian a un amplio rango que se procuró fuese representativo del espectro social, y tomando 
como criterio principal, aunque no exclusivamente, el académico. Es decir, fueron seleccionados partiendo de niveles de cualificación.

\section{Procedimiento}

Tras serle entregada a cada sujeto una baraja de dichas fichas, se les pidió que especificasen debajo, a poder ser en forma de listas, todo aquello que conociesen, como caracteristicas de las profesiones que les eran presentadas, y se les indicaba que trabajasen deprisa, pues no dispondrían más que de dos minutos para cada ficha. Se trabajó en horas de clase, en concreto en la de Ciencias Sociales, y previamente se les dieron cinco ejemplos como práctica.

\section{Resultados}

Hemos estructurado la información recogida para cada categoria conceptual - 20 en total - que representan determinadas profesiones - ver tabla I- en tres partes: una primera - tabla 2- donde se recoge la información por niveles de escolaridad, con la finalidad de poder comparar las modificaciones de los esquemas - sobre todo de su contenido- en función de la edad, pero recogiendo sólo las características o propiedades emitidas, al menos, por dos sujetos, que suponen un 8 por 100, ya que cada muestra se componía de 2 s sujetos, y ordenadas de mayor a menor frecuencia. A continuación - tabla 3- se ha analizado el contenido de dichas representaciones para intentar describir la estructura formal que subyace a las estructuras conceptuales -que a su vez subyacen a los enunciados lingüísticos-, representadas por variables y subvariables -acciones, objetos, propiedades, instrumentos, etc., ya que por la estructura de la tarea en si los agentes les fueron dados a los sujetos-. En tercer lugar - tabla 4-, se ha elaborado para algunas categorías un esquema, guión o estructura conceptual prototípica que sintetiza y agrupa el contenido de los tres niveles de edad, para simplicar la información, en una única representación conceptual, ordenándola en función de las frecuencias.

Es decir, resumiendo, se han determinado empíricamente los contenidos de estas categorias, para una muestra de sujetos determinada; se han presentado en forma de guión o esquema y se ha elaborado una estructura formal y unas reglas formales para análisis posteriores -fundamentación de predicciones $y$ expectativas- de dichas estructuras conceptuales. El resultado creemos que es analizable tanto desde el concepto de "frame" de Minsky (1972), como desde el "script» de Abelson (1981), o de Nelson (1973) o desde el prototipo de Rosch (1976), o esquema de Rumelhart (i 980), y por supuesto dentro del marco de los "social schemata" (Fiske y Taylor, 1984).

Finalmente la tabla, representa el esquema - vacio de contenido- que ejemplifica cómo se organiza o estructura la información para determinadas categorías sociales - tabla sa- y naturales y artefactuales $-s b-$. Los datos sobre los que se ha elaborado esta estructura son objeto de otro trabajo (Peraita, en prensa).

\section{Discusión}

La finalidad de nuestra recogida de datos ha sido determinar empíricamente el contenido de una serie de categorias -que en cuanto estructuras de conocimiento son vacías - para, partiendo de esos contenidos suministrados por los sujetos a través de una tarea eminentemente verbal y abierta - la única restricción era el tiempo de producción-, poder diseñar una estructura formal para las estructuras conceptuales que subyacen al lenguaje con el que los sujetos describen ciertos aspectos de la realidad que les rodea, en este caso categorías de personas - esquemas de roles. A partir de ese momento, podemos obtener reglas formales, para analizar las expresiones del lenguaje natural, y convertirlas en bases conceptuales, e incluso puede formalizarse una estructura de creencias. No olvidemos que el contenido de esquemas sociales, ya sean del tipo esquemas de personas, de roles, del propio yo o de acontecimientos, etc. (Fiske y Taylor, 1984; De Vega, 1984), en cuanto estereotipos llevan implícitas creencias, mejor dicho, son sistemas de creencias.

Esas reglas formales deben responder a las preguntas más usuales - terminales de Minsky - prototípicas, que uno se haría en una situación dada (texto-discur- 
so) o ante una persona o acontecimiento, y son imprescindibles para comprender un discurso, una situación, y a las personas y objetos que los componen. El número de preguntas prototípicas que «definen» una situación - persona, objeto, etc.- , sería problemático, y en cualquier caso, variable, móvil, flexible y dependiente del contexto (aunque podría ser de otro modo desarrolladas las implicaciones sobre los primitivos de signficado $\mathrm{y}$ los componentes planteada por Wilks, 1981). No son éstas preguntas que impliquen definición, puesto que el conjunto de valores $y / o$ variables de un concepto no son definicionales (Rumelhart y Ortony, 1977).

En nuestro caso hemos obtenido tres preguntas prototípicas básicas en torno a las cuales se organiza la información de roles sociales o profesiones para esa muestra de sujetos (ver tabla s A), y que invariablemente gira en torno a la categoría variable acción - lo que hacen las personas pertenecientes a una determinada profesión-, cualidad o atributos caracteristicos - cómo es- y medio o instrumentos necesarios para cumplir su papel. Una somera comparación con el esquema de preguntas o terminales de la tabla s B correspondiente a un trabajo sobre categorias naturales, presenta diferencias sustanciales.

El significado - y la representaciónque la serie de categorias que presentamos en este trabajo tienen para los niños es un significado cuya definición -como dice Minsky (1975) refiriéndose al significado de Child's Birthday, página $242-$, no puede encontrarse en ningún diccionario (quizá en uno elaborado a partir de las representaciones o conceptualizaciones de niños de esas edades, y además que fuese modular o sectorial), entre otras cosas porque la mayoría de esos conceptos o categorias están impregnados de un cierto sabor cultural inevitable -o más bien socio-cultural - con lo cual quiero decir que esas descripciones de categorías profesionales, obtenidas a partir de niños de un ámbito socio-cultural distinto, quizá presentarían otros matices. Por supuesto, también las de un grupo de adultos.

Aunque no vamos a prestarle aquí una atención explícita, un aspecto importante del conjunto de datos que presentamos es lo que podría llamarse «rectificación del esqueman, considerando como tal a las modalidades introducidas en la caracterización de profesiones por los niños de $4^{\circ}$ de EGB, con respecto a los de $2 .^{\circ}$ y por los niños de $7 .^{\circ}$ con respecto a los de los cursos anteriores. Esta modificación del esquema podría analizarse de acuerdo con parámetros cuantitativos -número de rasgos o características, frecuencias parciales y totales, etc.-, y cualitativos - contenidos de los esquemas en cada edad, variables y subvariables en torno a las cuales se agrupan, etc.- La aportación de un estudio de este tipo al tema de la formación y desarrollo de esquemas sería indudable.

\section{Resumen}

El presente trabajo establece una comparación, a nivel teórico, entre las caracteristicas de ciertas estructuras de conocimiento de la vida diaria que suelen abordarse por separado, y que una consideracion más pormenorizada de las mismas nos hace ver como muy semejantes.

Nos referimos, por una parte, al guion o script, tipo de esquema que representa bechos o sucesos, y, por otra, a las categorias naturales que representan objetos de la vida diaria. Se ha llevado a cabo un análisis técnico preferentemente estructural, seguido de uno empirico, sobre datos obtenidos de muestras de sujetos de tres cursos de EGB $\left(2 .^{\circ}, 4 .^{\circ} y 7 .^{\circ}\right)$.

La analogia se ba llevado a cabo sobre diferentes aspectos, en concreto, sobre la naturalezo esquemática de ambos tipos de representaciones, sobre los ejes sincrónico y diacrónico, estructura interna y organización jerárquica, procesos de formación $y$ desarrollo, etc.

El análisis de datos nos revela las unidades cognitivas o componentes cognitivos a que en ültimo lugar se reducen las caracterizaciones becbas por los sujetos de categorias naturales y sociales.

\section{Summary}

This paper aims at establishing a comparison, on a theoretical level, between the characteristics of a number of structures of knowledge of everyday life, wich are generally dealt witb separately, as individual structures, but which, after a more detailed consideration, seems to us to be very much alike.

Specifically we are referring on the band to the script, kind of scheme wich represents facts or events in everyday life, and, on the other band, to the natural categories wich represents pbysical objects and social categories. A theoretical, structural, analysis bas been made, followed by an empirical one, carried out using data from samples obtained among students of several EGB grades. 
The comparison bas been carried out on different aspects: on the sebematic nature of botb representation types, on the dyacronic and syncronic axes, inner structure and byerarcbical organization, formation and development processes, etc.

The data lesting shows the cognitive wnits or components to wich, in the last resort, the caracterizations of natural

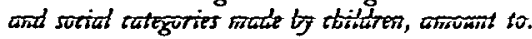

\section{Résumé}

Le present travail établit une comparaison, an niveau tbeiorique, entre des caracteristiques de quelques structures de la connaissäse de tous les jours que sont souvent envisagées independemment, mais que, si on les regarde de plus près, on les trowe tris semblables.

Nous faisons référence d une part au script, sorte de scbèma qui represente des faits, et done autre aux categories naturelles qui representent des objects tris familieres. On a mene un analyse theorique structurel, suivi d un autre empirique sur des donnees abtenues a partir de groupes des sujets des ecoles primaires. Une analogie a ite itablie sur la nature schematique de deus sortes de representations ainsi que sur les axes diacronique et sincronique, structure interne et organisation jérarchique, procès de developpement etc.

L'analyse des doneées met en evidence les composants cognitifs auxquels se reduisent les caracterisations faites par les sujets des categories naturelles et sociales.

\section{Referencias}

Abeison, R. P.: «Psychological Status of the Script Concept». American Psychologist. Vol. 36, 7, págs. $715-729,198 \mathrm{I}$.

ANDERSON, J. R.: Tbe arcbitecture of cognition. Harvard. Unpres, 1983.

ArnAu, J.: "Procedimiento de prosa y estructuras cognitivas». En MAYOR, J. (Ed.): Actividad bumana y procesos cognitivos. Alhambra Universidad, 1985 .

Barsaiou, L. W.: "Ad hoc categories". Memory and cognition. Vol. 11, 3, págs. $211-227,1983$.

Bower, G. H.; BıACK, J. B., y Turner, T. J.: "Scripts in memory for texts». Cognitive Psychology, 1 I, págs. II 7-220, 1979.

GAi.AmBOS, J. A., y Rips, L. J.: “Memory for routines». J.V.L.V.B. Vol. 21 , págs. 260-281, 1982.

MEdin, D. L., y SMiTH, E. E.: "Concepts and concepts formation". Annual Review of Psychology. Vol. 35 , págs. $113-138,1984$.

Mervis, C. B., y RoSCH, E.: «Categorization of Natural Objects». Annual Review of Psychology. 32, págs. 89-119, 1981.

MINSKY, M.: «A framework for representing knowledge». En WINSTON, H. W.: Tbe psychology of computer vision. Mc Graw Hill, 1975.

NEISON, K.: «How Children Represent Knowledge of Their World In and out of Language: A Preliminary Report». En SIEGIER, R. S. (Ed.): Children's Thinking. What develops? Lawrence Erlbaum Associates, Hillsdale, Nueva Jersey, 1978.

- "Social cognition in a script framework». En FI.AVEI.J., J., y Ross, L. (Eds.): Social cognitive development. Frontiers and possible futures. Cambridge University Press, 1981 .

- "The derivation of concepts and categories from events representations». En SCHOI.NICK, E. K.: New Trends in conceptual representation: Challenges to Piaget's Tbeory? LEA, 1983.

PerAit^, H.: «El nivel bàsic de categorització: un estudi comparatiu basat en l'analisi dels atributs de nou taxonomies". En estudi experimental del bilingüisme. Fundació Caixa de Pensions, Barcelona, 1984.

- "Análisis de la estructura de rasgos de categorias naturales, en una muestra de bilingües». (Fn prensa).

RosCh, E. H.: «Natural Categories». Cognitive Psycbology. 4, págs. 328-350, 1973.

ROSCH, E.: «Principles of categorization». En ROSCH y LioID (Eds.): Cognition and categorization. Lawrence Erlbaum, Associates, Hillsdale, Nueva Jersey, $197^{8}$.

ROSCH, F.., y MERvis, C. B.: «Family resemblances: studies in the internal structure of categories». Cognitive Psyschology. 7, págs. 575-605, 1975.

RumelharT, D. E.: «Schemața: The buildings blocks of cognition». En SPIRo et al (Eds.): Theoretical issues in reading comprebension. Lawrence Erlbaum Associates, Nueva Jersey, 1980.

RUmei.hART, D. E., y ORTONY, A.: "The representation of knowledge in memory". En ANDERSON, R. C.; SpIRO, R., y MONTAGue, W. F. (Fds.): Schooling and the acquisition of knowledge. Iawrence Frlbaum Associates, Hillsdale, Nueva Jersey, 1977.

SANTA CRuz, J.: «La representación del conocimiento». En MaYOR (Ed.).: Psicologia del pensamiento y del lenguaje. UNED, 1984 .

Schank, R. C.: "The structure of episodies in memory". En Bobrow, D. G., y Collins, A. (Eds.).: Representation and understanding. Studies in cognitive science. Academic Press, 1975.

SCHANk, R. C., y ABEISON, R. P.: Scripts, plans, goals and understanding. An inquiry into buman knowledge structures. Lawrence Erlbaum Associates. Nueva Jersey, 1977.

SCHOI.NICK, E. K.: "Why are new trends in conceptual representations: a challenge to Piaget's Theory?". En SCHOI.NiCK, E. K.: New Trends in conceptual representation: Cballenges to Piaget's Theory? LEA, 1983.

Veg^ DE, M.: Introducción a la Psicologia Cognitiva. Madrid, Alianza, 1984.

WII.KS, Y.: «A position note on natural language understanding and artificial intelligence». Cognition. Vol. 10, págs. $337-340,19^{81}$.

WINOGRAD, T.: "Fame representations and the declarative procedural controversy". En Bobrow y Coll.INS (Eds.): Representation and understanding. Studies in cognitive science. Nueva York, Academic Press, 1979 . 


\section{A. Apéndice}

\section{TABLA I}

Clasificación de las 20 profesiones en categorias, estructuradas según la cualificación profesional

TITULADOS SUPERIORES: Abogado, arquitecto, médico, químico.

TITULADOS MEDIOS: Delineante, enfermera, profesor ${ }^{1}$.

OBREROS CUALIFICADOS: Carpintero, fontanero, secretaria, zapatero.

OBREROS SIN CUALIFICAR: Albañil, agricultor, pescadero.

ARTISTAS: Bailarina, escritor, pintor.

OTROS (que no pueden agruparse según el esquema anterior): Deportista, militar, ama de casa.

1 Dado que los sujetos son de EGB bacen sinónimos o consideran profesor al maestro.

\section{TABLA II}

Rasgos descriptivos de cada categoria profesional, producidos por, al menos, dos sujetos, $y$ agrupados por niveles de edad. Cada uno va acompañado por su frecuencia absoluta y relativa.

\section{F. A. (\%)}

ABOGADO

2.० EGB

N. 25

Defiende a los delin-

cuentes ....... $19 \quad 72$

Ayuda a la gente ... 3 I 2

Llevan capas ...... 24

Van a los juicios ... $\quad 2 \quad 8$

$\begin{array}{lll}\text { Ayudan a los jueces. } & 2 & 8\end{array}$

4. ${ }^{\circ} \mathrm{EGB}$

N. ${ }^{\circ} 25$

Defiende a una.persona .......... 728

Defiende a los delincuentes ...... $4 \quad 16$

Arregla problemas . $3 \quad 12$

Es justo ....... 2

7.० EGB

N.' 25

Defiende a las personas ....... 2184

Buscan pruebas .... $3 \quad 12$

Divorcian ....... 2

Preparan preguntas . 28

Escribe ........ 28

\section{ARQUITECTO}

2. ${ }^{\circ} \mathrm{EGB}$

N.०25

Hace planos de casas. $21 \quad 84$

Usa regla, lápiz y goma ......... $6 \quad 24$

Hace dibujos ..... $4 \quad 16$

Usa mesa especial .. $3 \quad 12$

4. ${ }^{\circ} \mathrm{EGB}$

N. 25

Dibuja planos .... $9 \quad 36$

Es constructor .... $3 \quad 12$

Manda a los albañiles para que hagan co-
F. A. $(\%)$

F. A. $(\%)$
7. ${ }^{\circ}$ EGB

N. ${ }^{\circ} 25$

Dice cómo hacer una casa . . . . . . $12 \quad 4^{8}$

Hace planos ..... 9. 36

Diseña viviendas . . . $6 \quad 24$

\section{MEDICO}

2. $\mathrm{EGB}$

N. ${ }^{\circ} 25$

Cura gente ....... $18 \quad 72$

Receta medicinas ... I3 \$2

Pincha ........ $7 \quad 28$

Opera; da puntos .. $3 \quad 12$

Tiene bata blanca .. $3 \quad 12$

Usa algodón ..... 2 2 8

Ve a los niños ...... 2

Es importante .... 2.8

$4^{\circ}$ EGB

N.o 25

Cura a los enfermos. Is 60

Da jarabe y recetas . $6 \quad 24$

Inteligente ...... s 20

Amable ........ $4 \quad 16$

Trabajador ...... 28

7.० EGB

N. ${ }^{2}$ s

Cura enfermos ..... $16 \quad 64$

Opera ......... 1s 60

Receta ......... 9 96

Analiza ....... $4 \quad 16$

Mira ............ 4,16

Atiende enfermos .. $3 \quad 12$

Ayuda .......... $3 \quad 12$

Visita enfermos .... $3 \quad 12$

Arriesgado ....... 2

\section{QUIMICO}

2.0 EGB

N.०25

Mezcla líquidos ( $y$

hace) $\ldots \ldots \ldots$ is 60

Hace experimentos . $4 \quad 16$

Inventa cosas .... $3 \quad 12$
4. ${ }^{\circ} \mathrm{EGB}$

N.o 29

Inventa cosas para la humanidad .... 10 40

Es listo ....... $7 \quad 28$

Hace experimentos. 416

Mezcla líquidos .... 3 I 2

Es feo ......... $3 \quad 12$

Es bajo ........ 2

Es flaco $\ldots \ldots \ldots \ldots, 28$

$7 .^{\circ} \mathrm{EGB}$

N. ${ }^{2} 2$

Inventa fórmulas ... is 60

Experimenta ...... I I 44

Investiga $\ldots \ldots \ldots .7 \quad \mathbf{2 8}$

Observa ........ 5 20

Descompone ...... 3 I 12

Hace medicinas .... $3{ }_{3} 12$

Analiza ........ 20

\section{DELINEANTE}

2. ${ }^{\circ} \mathrm{EGB}$

N. 025

Hace dibujos ...... 20

Hace casas ....... 13 \$2

Tiene su mesa....., 20

Usa pincel, pinturas

y regla ........, 20

Hace planos ..... $3 \quad 12$

$\begin{array}{ll}\text { Ayuda al arquitecto . } & 2 \\ & 8\end{array}$

$4 .^{\circ} \mathrm{EGB}$

N. ${ }^{\circ} 29$

Dibuja casas ..... , 28

Hace lineas y casas . $\quad 2 \quad 8$

$70^{\circ} \mathrm{EGB}$

N.० 25

Hace planos ..... $19 \quad 72$

Dibuja .......... 936

Hace dibujos linea-

les ........ 624

Estudia matemáticas. $3 \quad 12$

Ayuda al arquitecto. $3 \quad 12$ 


\section{ENFERMERA}

2.0 EGB

N.0 25

Cura a los enfermos. $\quad 9 \quad 36$

Lleva bata blanca . . $7 \quad 28$

Opera ........ s 20

Trac la comida .... $4 \quad 16$

Usa algodón y zue-

$\cos \ldots \ldots \ldots \ldots 3_{12}^{\prime} 12$

Ayuda al médico... 3 iz

$44^{\circ} \mathrm{EGB}$

N." 25

Ayuda al doctor ... 14 ,6

Ayuda a las personas. 520

Es simpática ..... 4 16

Es guapa ....... $3 \quad 12$

Es inteligente .... $22^{2} 8$

Es rápida ....... 2

\section{$7 .^{\circ} \mathrm{EGB}$}

N. ${ }^{2}$ s

Ayuda al médico . . $17 \quad 68$

Cuida a los enfermos. $14 \quad 56$

Escribe recetas .... ; 20

Pone inyecciones... 24

Da de comer a los enfermos ......

\section{PROFESOR}

$2.0 \mathrm{EGB}$

N.०25

Enseña a multiplicar y dividir ...... $12 \quad 48$

Pinta en la pizarra.. $7 \quad 28$

Castiga ........ $4 \quad 16$

Usa pizarra y lápiz. 446

Corrige el cuaderno $2 \quad 8$

Da la lección ..... 2

4. ${ }^{\circ} \mathrm{EGB}$

N. 25

Enseña . . . . . . . $17 \quad 68$

Es inteligente .... 5 20

Es chillón ....... , 12

Educa ......... ; 20

Es guapo, castigón, amable, regañón. . 28

Sabe explicar ..... 2 8

\section{$70^{\circ} \mathrm{EGB}$}

N.o 25

Enseña ........23

Educa $\ldots \ldots \ldots \ldots .1144$

Explica las lecciones $7 \quad 28$

Regaña mucho ...., 20

Califica ........., 20

Pone muchos debe-

res $\ldots \ldots \ldots \ldots, 416$

Pone exámenes .... $4 \quad 16$

Enseña a ser algo en la vida ........

\section{CARPINTERO}

2. $\mathrm{EGB}$

N. ${ }^{2} 25$

Hace armarios, sillas, mesas ....... $12 \quad 48$

Hace cosas de made-

ra .......... 936

$\begin{array}{ccc}\text { Coge el martillo } \ldots . . . & 8 & 32\end{array}$

$44^{\circ} \mathrm{EGB}$

N. ${ }^{2} 2$

Hace cosas de made-

ra ........... 1040

Arregla muebles ... 8

Es rápido ...... 2

$\begin{array}{llll}\text { Usa herramientas . . } & 2 & 8\end{array}$

Trabaja la madera .. $2 \quad 8$

Fs fuerte $\ldots \ldots \ldots, \quad 2 \quad 8$

7. ${ }^{\circ} \mathrm{EGB}$

N. ${ }^{\circ} 29$

Hace muebles ..... 2 I 84

Arregla muebles ... $12 \quad 48$

Barniza ....... $6 \quad 24$

Hace objetos de madera $\ldots \ldots \ldots \ldots 6 \quad 64$

Sierra ........ , 20

Es mañoso $\ldots \ldots \ldots 2_{2} 8$

\section{FONTANERO}

2. EGB

N.o 25

Arregla grifos y tu-

berías ........ 1972

Usa herramientas .. 4416

4. ${ }^{\circ} \mathrm{EGB}$

N." 25

Arregla cañerías ... $18 \quad 72$

Es fuerte $\ldots \ldots \ldots 2_{2} 8$

Es listo $\ldots \ldots \ldots \ldots 2_{2} 8$

$7 .^{\circ} \mathrm{EGB}$

N.o 25

Arregla cañerías ... $18 \quad 72$

Pone tuberias ..... $18 \quad 72$

Pone grifos ....... 99

Monta bañeras . . . $4 \quad 16$

Desatasca ........ $4 \quad 16$

Suelda ......... $3 \quad 12$

Pone redes de cañe-

rías ......... 2

\section{SECRETARIA}

2. EGB

N.o 25

La secretaria escribe a máquina ..... 15 60

$\begin{array}{lll}\text { Usa bolígrafo o lápiz. } & 12 & 48\end{array}$

Hace cosas al jefe .. $8 \quad 3 \quad 32$

Trabaja en la oficina. $\quad 3 \quad 12$

$\begin{array}{ll}\text { Arregla papeles .... } & 2 \\ & 8\end{array}$

Hace cuentas, etcéte-

\section{$44^{\circ} \mathrm{EGB}$}

N. ${ }^{\circ} 25$

La secretaria trabaja

en la oficina ... $\quad 7 \quad 28$

Da cartas al director. $3 \quad 12$

Escribe a máquina . 312

Es vieja ........ $3 \mathbf{1}_{2}$

Es amable ...... $3 \quad 12$

$70^{\circ} \mathrm{EGB}$

N.0 25

Escribe a máquina . 14

Ayuda al jefe ..... $12 \quad 48$

Copia......... \& 20

Llama por teléfono $s 20$

Escribe cartas .... s 20

Sabe taquigrafia .... , 20

Da recados ...... $3 \quad 13$

Redacta ........ 3

\section{ZAPATERO}

2. ${ }^{\circ}$ EGB

N. ${ }^{\circ} 25$

Arregla zapatos .... $17 \quad 68$

Hace zapatos ..... $7 \quad 28$

Usa cuero, hebillas . $7 \quad 28$

$\begin{array}{rrrr}\text { Vende zapatos ..... } & 2 & 8\end{array}$

Cose zapatos ..... $2 \quad 8$

$4 .^{\circ} \mathrm{EGB}$

N. ${ }^{\circ} 29$

Arregla zapatos . . . $17 \quad 68$

Sabe coser ....... $4 \quad 16$

Hace zapatos ....... 4 4 16

Es rápido $\ldots \ldots \ldots 2^{2} 8$

7.० EGB

N. ${ }^{\circ} 25$

- Remienda ....... $16 \quad 64$

Pone suelas ...... $8 \quad 32$

$\begin{array}{lll}\text { Hace zapatos ..... } 7 & 7 & \mathbf{2} 8\end{array}$

Pone tapas ...... 24

\section{ALBAÑIL}

2.० EGB

N. 25

Hace casas ....... $16 \quad 64$

Usa cemento y cubo. $12 \quad 4^{8}$

Pone ladrillos ..... I 1144

$\begin{array}{rrr}\text { Hace colegios ..... } & 2 & 8\end{array}$

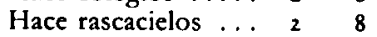

$4^{\circ} \mathrm{EGB}$

N.o 25

Hace casas ....... $18 \quad 72$

Es fuerte $\ldots \ldots \ldots 4416$

Es trabajador $\ldots \ldots 2^{2} \quad 8$

Coge ladrillos y los

pega con cemento $2 \quad 8$

Es alto $\ldots \ldots \ldots \ldots 3 \quad 12$

$70^{\circ} \mathrm{EGB}$

N. 025

Hace casas ....... 14,56

Pone tejas ........ 9 


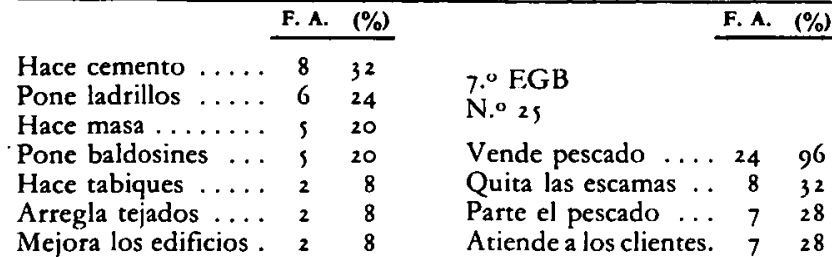

\section{AGRICULTOR}

\subsection{FGB \\ N.० 25}

Trabaja en el campo $12 \quad 4^{8}$

Siembra (tomates, lechugas) $\ldots \ldots \ldots 9936$

Hace surcos ...... $8 \quad 32$

Trabaja con el tractor, hoz, semillas. $6 \quad 24$

Planta árboles .... $3 \quad 12$

\section{4. $\mathrm{EGB}$ \\ N.o 25}

Siembra comida ... $8 \quad 32$

Es trabajador .... $7 \quad 28$

Trabaja en el campo. s 20

Es alto ......... s 20

Fuerte ......... 5 16

Recoge ........ $4 \quad 16$

Es bajo ........ $3 \quad 12$

Es sucio ........ 2. 8

Compra instrumentos $\ldots \ldots \ldots \ldots 28$

7.० EGB

N.o 25

Cuida la tierra ..... 13 \$2

Cultiva la tierra .... 9936

Cosecha ....... 8

Sieğa $\ldots \ldots \ldots \ldots 728$

Recoge el fruto ... $6 \quad 24$

Planta ........ , 20

Vende los frutos ... $3 \quad 12$

Labra la tierra ..... $3 \quad 12$

\section{PESCADERO}

2. $0^{\circ} \mathrm{EGB}$

N.० 25

Cazan pececitos .... $10 \quad 40$

Venden pescado .... 936

Usan redes ....... 8

Venden calamares...

Usan gusanos .... $3 \quad 12$

Se van en barco ... $3 \quad 12$

\section{4. ${ }^{\circ} \mathrm{EGB}$}

N.o 25

Pescan peces $\therefore . .$. is 60

Da pescado a la gen-

te .......... ; 20

Fuerte ....... 3 I 2

Delgado ........ 2

Moreno ......... 2

Valiente ........ 2

Gustoso del mar ... 2.2

Guapo ......... 2

\section{BAILARINA \\ 2. ${ }^{\circ} \mathrm{FGB}$ \\ $4 .^{\circ} \mathrm{EGB}$ \\ N. ${ }^{\circ} 25$}

N. ${ }^{2} 25$

Baila ......... $16 \quad 64$

Lleva traje blanco y corto ........ 9 36

I.leva zapatillas .... ; 20

4. $\mathrm{EGB}$

N. ${ }^{2} 5$

Baila ........ 14, \$6

Es finita (delgada) . $4 \quad 16$

Es ágil ........ 3 I 2

Simpática ....... 3

Linda . . . . . . 2

7.० $\mathrm{EGB}$

N. 25

Baila $\ldots \ldots \ldots \ldots 19 \quad 76$

Hace gimnasia .... $6 \quad 24$

Aprende música ... $4 \quad 16$

Se pone de puntas . 312

Se pone trajes .... $3 \quad 12$

Da clases ....... $3 \quad 12$

Fs artista ....... 2.8

Mueve los pies .... 2

Hace funciones .... 2

Distrae ........ 28

\section{ESCRITOR}

2. $\mathrm{EGB}$

N. 25

Escribe ........ I $2 \quad 48$

Escribe novelas .... 1144

Escribe cuentos .... $11 \quad 44$

Escribe poesia .... 4 I6

Usa lápiz y papel .. 4 I6

4. EGB

N. 25

Escribe ........ 2 I 84

Escribe novela ...., 20

Escribe cuentos.... , 20

Escribe poesia ..... 3 I 2

Escribe libros ..... $6 \quad 12$

Escribe teatro ..... I 4

7.० EGB

N. ${ }^{\circ} 2$

Escribe libros ..... $21 \quad 84$

Escribe cosas ..... Io 40

Redacta ........., 20

Piensa ......... $4 \quad 16$

Lee mucho ....... 4 I6

Escribe poesia .... $3 \quad 12$

Es culto ........ $3 \quad 12$

Escribe articulos ... $22^{2} 8$

\section{PINTOR}

$20^{\circ} \mathrm{EGB}$

N.० 25

Pinta cuadros .... $19 \quad 76$

Usa pincel ....... $66^{6} \quad 24$

Pinta las paredes ... $3 \quad 12$

Hace cuadros ..... $12 \quad 48$

Pinta y dibuja ..... , 20

Pinta paredes ..... 3

$70^{\circ} \mathrm{EGB}$

N. ${ }^{2} 25$

Hace cuadros ..... Is 60

Fs un artista ..... 8

Pinta personas ..... $6 \quad 6$

Mezcla colores ... $6 \quad 24$

Pinta paisajes ....., s 20

Dibuja ......... $3 \quad 12$

Copia ......... $3 \quad 12$

Transmite lo que siente $\ldots \ldots \ldots 2 \quad 8$

\section{DEPORTISTA}

$2 .{ }^{\circ} \mathrm{EGB}$

N. ${ }^{\circ} 25$

Hacen carreras con

bicis ......... $12 \quad 48$

Van en bicicleta ... 111

Dan pedales ...... $6 \quad 24$

Dos ruedas ...... 3 I 2

Hacen deporte..... 3 , 12

Ganan algunas veces. 28 .

Corren por la carre-

tera $\ldots \ldots \ldots \ldots \quad 2 \quad 8$

$4 .^{\circ} \mathrm{EGB}$

N. 029

Practica deporte ... II 44

Corre mucho ..... 3,12

Es fuerte $\ldots \ldots \ldots{ }_{2} 8$

7.० EGB

N.o 25

Entrena ....... 14, 56

Corre ......... 1248

Hace gimnasia .... $7 \quad 28$

Salta.......... s 20

Juega al fútbol .... , 20

Juega al tenis ..... ; 20

Hace deporte ...... $3 \quad 12$

Pelea de forma amis-

tosa $\ldots \ldots \ldots \ldots$, 12

Compite....... 3

\section{MILITAR}

2.० EGB

N.o 25

Está en la mili .... $9 \quad 36$

Hace guerras ...... 8

Tiene escopeta ... 936

Uniforme ....... 3 


\begin{tabular}{|c|c|c|c|c|c|c|c|c|}
\hline & F. A. & $(\%)$ & & F. A. & $(\%)$ & & F. A. & $(\%)$ \\
\hline $\begin{array}{l}\text { Hace ejercicio ..... } \\
\text { Mata a la gente .... } \\
\text { Aprende a coger ar- } \\
\text { mas } \ldots \ldots \ldots \ldots\end{array}$ & $\begin{array}{l}j \\
2\end{array}$ & $\begin{array}{r}12 \\
8\end{array}$ & $\begin{array}{l}\text { Se prepara para la } \\
\text { guerra } \ldots \ldots \ldots \ldots \\
\text { Hace ejercicio de ti- } \\
\text { ro } \ldots \ldots \ldots \ldots \ldots\end{array}$ & 10 & $\begin{array}{l}40 \\
20\end{array}$ & $\begin{array}{l}\text { Usa cristasol y leiía. } \\
4^{\circ} \text { EGB } \\
\text { N. }{ }^{\circ} 25\end{array}$ & 3 & 12 \\
\hline $\begin{array}{l}44^{\circ} \mathrm{EGB} \\
\mathrm{N} \cdot{ }^{\circ} 25\end{array}$ & & & $\begin{array}{l}\text { Aprende las ordenan- } \\
\text { zas } \ldots \ldots \ldots \\
\text { Viste uniforme } \ldots \ldots \\
\text { Hace guardias } \ldots \ldots\end{array}$ & $\begin{array}{l}3 \\
2 \\
2\end{array}$ & $\begin{array}{r}12 \\
8 \\
8\end{array}$ & $\begin{array}{l}\text { Hace cosas de la casa. } \\
\text { Es buena mujer . . . } \\
\text { Es rápida ........ }\end{array}$ & $\begin{array}{r}12 \\
4 \\
2 \\
2\end{array}$ & $\begin{array}{r}48 \\
16 \\
8 \\
8\end{array}$ \\
\hline $\begin{array}{l}\text { Hace la mili ....... } \\
\text { Va a la guerra ..... } \\
\text { Defiende la patria } \\
\text { Fs valiente } \ldots \ldots \ldots \\
\text { Robusto ......... }\end{array}$ & $\begin{array}{l}5 \\
5 \\
5 \\
3 \\
2\end{array}$ & $\begin{array}{r}24 \\
20 \\
20 \\
12 \\
8\end{array}$ & Salta y corre ..... & 2 & 8 & $\begin{array}{l}\text { 7.० F.GB } \\
\text { N.० } 25\end{array}$ & 2 & \\
\hline $\begin{array}{l}\text { Cumple órdenes } \ldots \\
\text { Es desordenado } \ldots \\
\text { Protege } \ldots \ldots \ldots\end{array}$ & $\begin{array}{l}2 \\
2 \\
2\end{array}$ & $\begin{array}{l}8 \\
8 \\
8\end{array}$ & $\begin{array}{l}20^{\circ} \text { F.GB } \\
\text { N.०25 }\end{array}$ & & & $\begin{array}{l}\text { Limpia } \ldots \ldots \ldots \\
\text { Hace la comida } \ldots \\
\text { Lava } \ldots \ldots\end{array}$ & $\begin{array}{l}22 \\
15 \\
12\end{array}$ & $\begin{array}{l}88 \\
60 \\
48\end{array}$ \\
\hline $\begin{array}{l}77^{\circ} \mathrm{EGB} \\
\text { N.*25 }\end{array}$ & & & $\begin{array}{l}\text { Limpia } \ldots \ldots \ldots \ldots \\
\text { Cocina } \ldots \ldots \ldots \ldots \\
\text { Friega } \ldots \ldots \ldots \ldots \\
\text { Barre } \ldots \ldots \ldots \ldots\end{array}$ & $\begin{array}{r}14 \\
12 \\
10 \\
6\end{array}$ & $\begin{array}{l}56 \\
48 \\
40 \\
24\end{array}$ & $\begin{array}{l}\text { Plancha } \ldots . . . . \\
\text { Cuida a sus hijos ... } \\
\text { Hace la compra .... } \\
\text { Cuida de la casa ... }\end{array}$ & $\begin{array}{l}12 \\
12 \\
11 \\
11\end{array}$ & $\begin{array}{l}4^{8} \\
4^{8} \\
44 \\
44\end{array}$ \\
\hline Defiende su patria. & 16 & 64 & Cuida a los hijos ... & 4 & 16 & Ayuda a su marido. & - 4 & 16 \\
\hline
\end{tabular}

\section{TABLA III}

Variables y subvariables que organizan la información para cada categoria profesional, pero sin considerar ya los niveles de edad $\left(20^{\circ}, 4 .^{\circ}\right.$ y $\left.7 .^{\circ} E G B ; N=75\right)$.

\section{ABOGADO}

Defiende

Ayuda

Busca

Arregla

Prepara

Divorcia

$\mathbf{V a}$

Escribe

¿COMO ES?

Justo
¿QUE HACE?

\section{QUIMICO}

¿QUE HACE?

$\begin{array}{ll}\text { Mezcla } & \begin{array}{l}\text { líquidos. } \\ \text { fórmulas. } \\ \text { cosas para la humanidad. } \\ \text { experimentos. }\end{array} \\ \text { Hace } & \end{array}$

Experimenta

Investiga

Observa

Descompone

Hace

Analiza

¿COMO ES?

Es

listo.

feo.

bajo.

flaco.

¿QUE USA?

Líquidos.

¿QUE USA?

Regla, lápiz, goma.

Mesa especial. planos.

dibujos.

Dibuja planos.

Diseña viviendas.

Dice

cómo hacer una casa.

Manda
a los albañiles que hagan cosas.

¿QUE ES?

Constructor.

Planificador de casas.

¿QUE HACE?

\begin{tabular}{|c|c|}
\hline Hace & $\begin{array}{l}\text { planos de casas. } \\
\text { planos. }\end{array}$ \\
\hline Dibu & $\begin{array}{l}\text { dibujos. } \\
\text { planos. }\end{array}$ \\
\hline Diseña & viviendas. \\
\hline $\begin{array}{l}\text { Dice } \\
\text { Manda }\end{array}$ & $\begin{array}{l}\text { cómo hacer una casa. } \\
\text { a los albañiles que hagan } \\
\text { cosas. }\end{array}$ \\
\hline
\end{tabular}

¿QUE HACE?

$\begin{array}{ll}\text { Cura } & \begin{array}{l}\text { gente. } \\ \text { enfermos. } \\ \text { niños. }\end{array}\end{array}$

\section{MEDICO}

¿COMO ES?

Es

arriesgado.

importante.

inteligente.

amable.

trabajador. 
TABLA III (cont.)

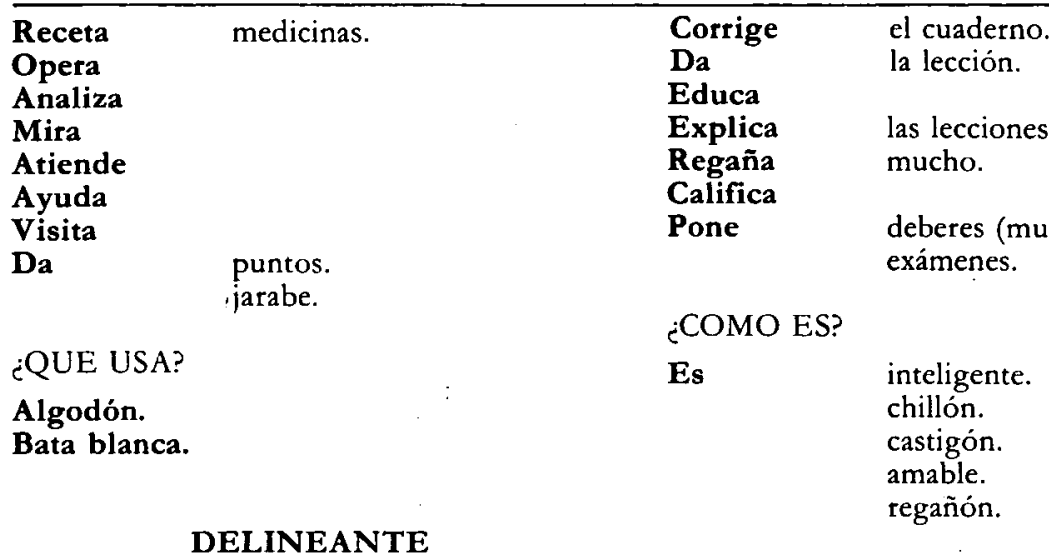

¿QUE HACE?

$\begin{array}{ll}\text { Hace } & \begin{array}{l}\text { dibujos -lineales-- } \\ \text { planos. } \\ \text { casas y líneas. }\end{array} \\ \text { Dibuja } & \text { casas. } \\ \text { Ayuda } & \text { al arquitecto. } \\ \text { Estudia } & \text { matemáticas. }\end{array}$

Mesa.

Pincel.

Pinturas.

Regla.
¿QUE USA?

\section{SECRETARIA}

¿QUE HACE?

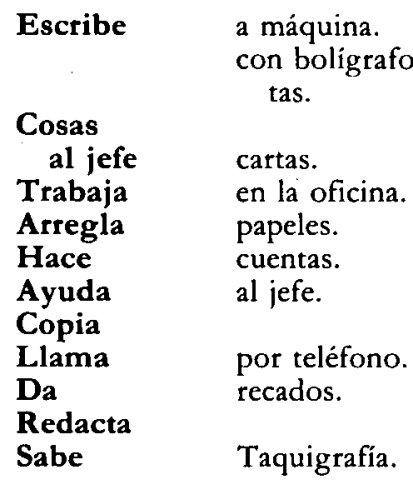

¿COMO ES?

¿QUE HACE?

$\begin{array}{ll}\text { Ayuda } & \text { al médico }- \text { o doctor- } \\ \text { Cuida } & \text { a los enfermos. } \\ \text { Ayuda } & \text { a las personas. } \\ \text { Cura } & \text { a los enfermos. } \\ \text { Opera } & \\ \text { Escribe } & \text { recetas. } \\ \text { Lleva } & \text { la comida. } \\ \text { Pone } & \text { inyecciones. } \\ \text { Da } & \text { de comer a los enfermos. }\end{array}$

¿COMO ES?

Es

\section{¿QUE USA?}

Bata blanca.

Algodón.

Zuecos.

\section{PROFESOR}

¿QUE HACE?

Enseña

a multiplicar.

a dividir.

a ser algo en la vida.
Es

vieja.

amable.

¿QUE USA?

Máquina.

Bolígrafo, Lápiz.

Cartas.

\section{ZAPATERO}

¿QUE HACE?

$\begin{array}{ll}\text { Arregla } & \text { zapatos. } \\ \text { Remienda } & \\ \text { Hace } & \text { zapatos. } \\ \text { Pone } & \text { suelas. } \\ & \text { tapas. } \\ \text { Vende } & \text { zapatos. } \\ \text { Cose } & \text { zapatos. } \\ \text { Sabe } & \text { coser. }\end{array}$

¿COMO ES?

Es

rápido.
Pinta
Castiga
en la pizarra.
Cuero.
Hebillas.

¿QUE USA? 


\section{CARPINTERO}

¿QUE HACE?

Hace

armarios, sillas, mesas. cosas de madera. muebles.

Arregla

Coge

Barniza objetos de madera.

Sierra

Trabaja la madera.

¿COMO ES?

Es

mañoso.

rápido.

fuerte.

¿QUE USA?

Madera.

Martillo.

Herramientas.

\section{FONTANERO}

¿QUE HACE?

$\begin{array}{ll}\text { Arregla } & \text { cañerías. } \\ \text { Prifos, tuberias. } \\ \text { tuberías. } \\ \text { grifos. } \\ \text { redes de cañerias. }\end{array}$

\section{Monta}

bañeras.

Desatasca

Suelda

¿COMO ES?

Es

fuerte.

listo.

¿QUE USA?

Herramientas.

\section{AGRICULTOR}

¿QUE HACE?

\section{Trabaja}

Siembra

Cuida

Hace

Cosecha

Siega

\section{Recoge}

Planta

Vende

Labra

Compra

¿COMO ES?

Es en el campo.

tomate, lechuga, comida.

la tierra.

surcos.

el fruto.

árboles.

los frutos.

la tierra.

instrumentos.

trabajador.

alto.

fuerte.

bajo.

sucio.
¿QUE USA?

Tractor, hoz, semillas.

Instrumentos.

\section{ALBAÑIL}

¿QUE HACE?

Hace

casas.

colegios.

rascacielos.

cemento.

masa.

tabiques.

Pone

Coge

Arregla

Mejora

¿COMO ES?

Es

ladrillos.

tejas.

baldosines.

ladrillos y los pega con cemento.

tejados.

edificios.

fuerte.

alto.

trabajador.

¿QUE USA?

Cemento y cubo.

\section{ESCRITOR}

¿QUE HACE?

Escribe

novelas.

cuentos.

poesía.

libros.

teatro.

Redacta

artículos.

Piensa

Lee

Ingenia

(mucho).

cosas.

¿COMO ES?

Es culto.

¿QUE USA?

Lápiz y papel.

\section{PINTOR}

¿QUE HACE?

Pinta

cuadros.

personas.

paisajes.

las paredes.

Hace cuadros.

Mezcla colores.

Dibuja

Copia

Transmite lo que siente. 
Es un artista.

¿QUE USA?

Pincel.

\section{BAILARINA}

¿QUE HACE?

Baila

Hace gimnasia.

Aprende música.

Se pone de puntas.

Da

Mueve

Hace

Distrae

¿COMO ES?

Es

\section{PESCADERO}

¿QUE HACE?

Vende

Pesca

Caza

Da

Usa

Quita

Parte

Atiende

Se va

¿COMO ES?

Es

pescado y calamares.

peces.

pececitos.

pescado a la gente.

redes.

gusanos.

las escamas.

el pescado.

a los clientes.

en barco.

fuerte.

delgado.

moreno.

etc.

¿QUE USA?

Redes.

Gusanos.
¿QUE HACE?

\section{Guerras}

Ejercicio

de tiro. salta y corre.

\section{Mata}

Aprende manejo de armas. ordenanzas.

Defiende

Protege

Guardias.

¿COMO ES?

Es

valiente.

robusto.

obediente.

desordenado.

¿QUE USA?

Tiene uniforme.

\section{AMA DE CASA}

¿QUE HACE?

Hace

Limpia

Lava

Plancha

Cuida a los niños.

de la casa.

Friega

Barre

Hace la compra.

Ayuda

al marido.

¿COMO ES?

Es

buena mujer.

rápida.

trabajadora.

¿QUE USA?

Cristasol y lejia.

\section{DEPORTISTA}

¿QUE HACE?

Entrena

Corre

Practica

Hace

Salta

Juega

Pelea

Compite

gimnasia.

¿COMO ES?

'Podria considerarse como rasgo clasificatorio o
taxonómico, que respondiera a la pregunta ¿QUE ES? al fútbol, al tenis.

de forma amistosa. 


\section{Algunos esquemas prototipicos para categorias profesionales}

El agricultor trabaja en el campo; siembra (tomates, lechugas, etc.); cuida la tierra; hace surcos; planta árboles; cosecha; recoge el fruto; vende los frutos; labra la tierra; compra instrumentos. Es trabajador, alto, fuerte... Usa diversos instrumentos (tractor, hoz, semillas).

El fontanero arregla cañerías, grifos y tuberias. Pone tuberias, grifos y redes de cañerías. Monta bañeras; desatasca y suelda. Usa herramientas. Es fuerte y listo.

El carpintero hace muebles, objetos (cosas) de madera - armarios, sillas, mesas-. Arregla muebles; usa el martillo y herramientas para trabajar la madera; barniza, sierra. Es mañoso, rápido y fuerte.

El quimico hace experimentos (o experimenta); mezcla liquidos; inventa fórmulas y cosas para la humanidad; investiga, observa, descompone, hace mediciones y analiza. Es listo; feo; bajo; flaco. Trabaja con líquidos.

El abogado defiende a los delincuentes y a las personas; ayuda a la gente y a los jueces; busca pruebas; arregla problemas; prepara preguntas; divorcia y va a los juicios. Escribe. Es justo.

\section{TABLA sA}

Esquema vacio de contenido que organiza la información para categorias de profesiones en niños de EGB.

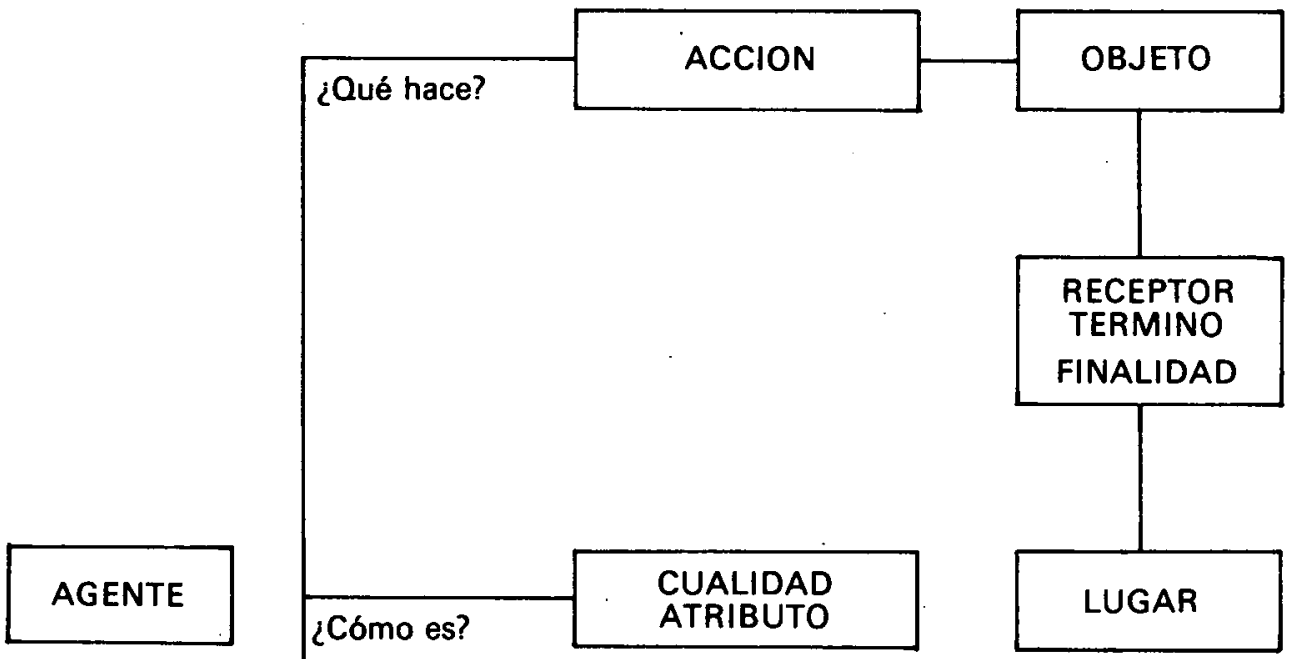


TABLA $; B$

Esquema que organiza la información para categorias naturales y artefactuales.

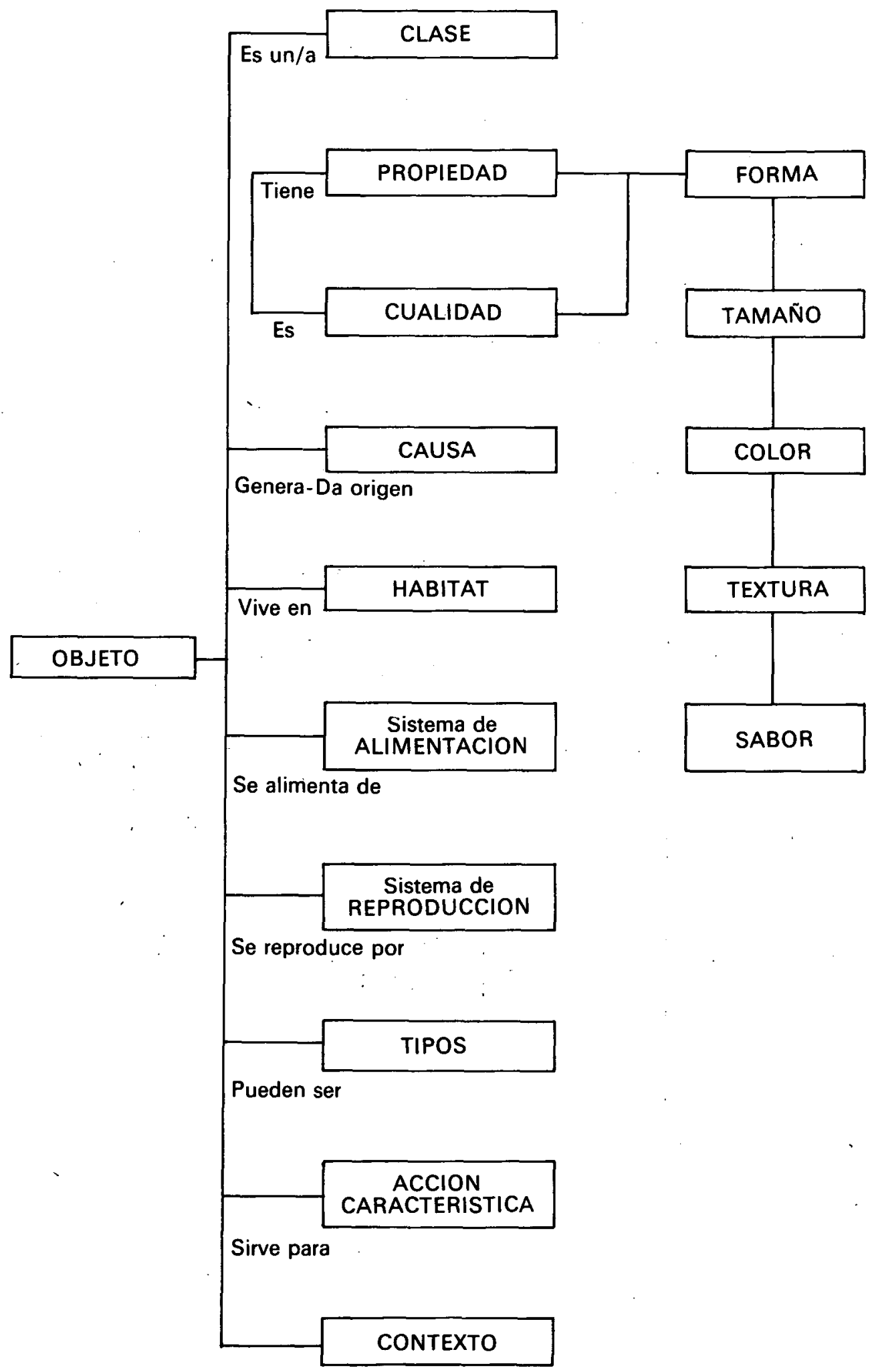

